CERN-TH.6987/93

ANL-HEP-CP-93-63

August, 1993

\title{
Bottom Quark Cross Sections at Collider and Fixed-Target Energies at the SSC and LHC
}

\author{
Edmond L. Berger ${ }^{a, b}$ and Ruibin Meng $^{a}$ \\ ${ }^{a}$ High Energy Physics Division \\ Argonne National Laboratory, Argonne, IL 60439, USA \\ ${ }^{b}$ CERN, Geneva, Switzerland
}

\begin{abstract}
Calculations of inclusive cross sections for the production of bottom quarks in proton-proton collisions are presented as a function of energy, transverse momentum, and Feynman $x_{F}$ for values of $\sqrt{s}$ from $100 \mathrm{GeV}$ to $40 \mathrm{TeV}$. In addition, we provide simple parametrizations of our theoretical results that should facilitate estimates of rates, acceptances, and efficiencies of proposed new detectors.
\end{abstract}

* To be published in the Proceedings of the Workshop on B Physics at Hadron Colliders, Snowmass, Colorado, June 1993.

CERN-TH.6987/93

August, 1993 
Calculations of heavy flavor cross sections at the planned energies of future hadron colliders assist in the design of experiments and in the evaluation of the merits of various options, such as experiments in fixed target modes and/or with detection concentrated in forward or central regions of phase space. In this paper, calculations are presented of inclusive cross sections for bottom quark production at energies from $\sqrt{s}=100 \mathrm{GeV}$ to $40 \mathrm{TeV}$. For several energies, cross sections are displayed as functions for transverse momentum $\left(p_{T}\right)$ for selected values of Feynman $x_{F}$. In addition, we provide simple analytic parametrizations of our theoretical predictions that should make our results easy to use in studies of expected acceptances and efficiencies of proposed new detectors. The theoretical computations are based on next-to-leading order QCD hard-scattering cross sections ${ }^{1,2}$ and the latest two-loop evolved parton densities obtained from a global fit of data from deep-inelastic lepton scattering and other reactions ${ }^{3}$. The work presented here is an update of an earlier publication ${ }^{4}$ to which we refer for the theoretical formalism and summary of its limitations.

The heavy quark inclusive cross section in perturbative QCD is obtained as a convolution of parton densities $f_{i / h}(x, \mu)$ with a hard-scattering cross section $\hat{\sigma}_{i j}\left(\hat{s}, M_{Q}, \mu, \alpha_{s}(\mu)\right)$. The heavy quark mass is $M_{Q} ; \hat{s}$ is the square of the parton-parton center-of-mass energy, $\hat{s}=x_{1} x_{2} s$; and $\mu$ is the renormalization/factorization scale that serves to separate long- and short-distance effects.

$$
\sigma\left(s, M_{Q}^{2}\right)=\int_{0}^{1} d x_{1} \int_{0}^{1} d x_{2} f_{i / h_{1}}\left(x_{1}, \mu\right) f_{j / h_{2}}\left(x_{2}, \mu\right) \hat{\sigma}_{i j}\left(\hat{s}, M_{Q}, \mu, \alpha_{s}(\mu)\right) .
$$

For the total cross sections reported here and for the cross sections differential in $p_{T}$, we use the scale choice $\mu=\sqrt{M_{b}^{2}+p_{T}^{2}}$. We adopt $M_{b}=4.75 \mathrm{GeV}$. Poor knowledge of the gluon density is a principal source of uncertainty for predictions of bottom quark cross sections at collider energies. In earlier work, we showed that measurements of the bottom quark cross sections by the CERN UA1 and FNAL CDF collaborations provide valuable constraints on the gluon density at intermediate values of Bjorken $x^{5}$. For LHC and SSC energies, the values of $x$ of interest extend over the range $10^{-6}$ to $10^{-2}$, or so. To explore the uncertainties associated with relative ignorance of the gluon density in this range, we adopt for part of our study the "singular" and "regular" gluon density parametrizations of the MRS collaboration. These are denoted, respectively, MRS $D_{-}^{\prime}$ and MRS $D_{o}^{\prime}$.

In Fig. 1 we show calculations of the inclusive cross section $\sigma(p p \rightarrow b \bar{b} X)$ obtained from an integration of the inclusive yield over all rapidity and transverse momentum. For 
the energy range $100 \mathrm{GeV}<\sqrt{s}<2 \mathrm{TeV}$ shown in Fig. 1 (a), the singular and regular parametrizations of the gluon density yield similar results. The differences become increasingly significant as $\sqrt{s}$ increases above $\sqrt{s}=2 \mathrm{TeV}$, as shown in Fig. 1 (b).

A $20 \mathrm{TeV}$ proton beam incident on a fixed proton target provides a center-of-mass energy $\sqrt{s}=200 \mathrm{GeV}$. For this SSC fixed-target option, we calculate $\sigma(b \bar{b} X)=1.27 \mu b$ for the singular set $D_{-}^{\prime}$ and $1.56 \mu b$ for the regular set $D_{o}^{\prime}$. Including uncertainties associated with variations of the choice of scale $\mu$ and b quark mass $M_{b}$, we estimate

$$
\sigma(b \bar{b} X, \sqrt{s}=200 \mathrm{GeV})=1.0 \text { to } 2.0 \mu b
$$

At $\sqrt{s}=40 \mathrm{TeV}$, the calculations yield $\sigma(b \bar{b} X) \simeq 0.45 \mathrm{mb}$ for set $D_{o}^{\prime}$ and nearly $2 \mathrm{mb}$ for set $D_{-}^{\prime}$. However, at this energy, gluon resummation effects ${ }^{6}$ are expected to be very significant, providing enhancement factors ${ }^{6}$ of $\sim 4$ for a regular gluon starting distribution $(x G(x) \rightarrow$ constant $)$ and $\sim 1.5$ for a singular distribution $\left(x G(x) \sim x^{-\frac{1}{2}}\right)$. Using these factors, we may multiply our $O\left(\alpha_{s}^{3}\right)$ results at $\sqrt{s}=40 \mathrm{TeV}$, obtaining $\simeq 1.8 \mathrm{mb}$ for set $D_{-}^{\prime}$ and $\simeq 3 m b$ for set $D_{o}^{\prime}$. Including estimates of other uncertainties, we quote

$$
\sigma(b \bar{b} X, \sqrt{s}=40 \mathrm{TeV})=1 \text { to } 3 m b
$$

Similar reasoning leads to an estimate appropriate at the LHC energy $\sqrt{s}=15.4 \mathrm{TeV}$ :

$$
\sigma(b \bar{b} X, \sqrt{s}=15.4 T e V)=0.5 \text { to } 0.9 m b
$$

In Fig. 2, we present the QCD order $\alpha_{s}^{3}$ differential cross section $d \sigma / d x_{F} d p_{T}^{2}$ as a function of $p_{T}$ for the SSC collider energy, for three values of Feynman $x_{F}$. For these results, we use the regular gluon density MRS $D_{o}^{\prime}$. In our earlier paper ${ }^{4}$, we showed that the influence of the more singular gluon density is felt most strongly at small $p_{T}\left(p_{T} \leq 25 \mathrm{GeV}\right)$ at $\sqrt{s}=40 \mathrm{TeV}$. Our theoretical results are shown as the solid curves in Fig. 2. Notable in Fig. 2 is the dramatic decrease in the cross section at $p_{T}=0$ by more than four orders of magnitude when $x_{F}$ is increased from 0. to 0.25. Comparison of Figs. 2 (b) and 2 (c) shows that this large drop is followed by a less remarkable decrease by a factor of 30 or so as $x_{F}$ is increased from 0.25 to 0.5 . At small $x_{F}$ and small $p_{T}$, the cross section is sensitive to the small $x$ behavior of the gluon density. In our previous paper, we provided calculations of rapidity and pseudo-rapidity distributions ${ }^{4}$. 
The theoretical results shown in Fig. 2 may be fitted with a fairly simple analytic expression. The form we adopted is

$$
\frac{d \sigma}{d p_{T}^{2} d x_{F}}=\frac{1}{\left(p_{T}^{2}+m_{b}^{2}\right)^{2}} \exp \left(A+B p_{T}\right)
$$

We treat quantities $A, B$, and $m_{b}$ as three free parameters whose values vary with $\sqrt{s}$ and $x_{F}$. The histograms in Fig. 2 show the results of our fits to the theoretical calculations. Fitted values of the three parameters are provided in Table I.a. The fitted value of our parameter $m_{b}$ is close to the value we used for the physical bottom quark mass $M_{b}$ in our calculation, to be anticipated since $<p_{T}>$ is approximately $M_{b}$, but no great significance should be attached to the fact that our fitted $m_{b}$ varies somewhat with $x_{F}$ and $\sqrt{s}$. The good agreement of the simple fit with the full theoretical calculation should make the fitted expression useful for estimates of rates, acceptances, and efficiencies.

Table I.a Fitted Parameters for $d \sigma / d p_{T}^{2} / d x_{F}$ for: $0<p_{T}<50 \mathrm{GeV}$ at SSC Collider Energy.

\begin{tabular}{|l|c|c|c|}
\hline$x_{F}$ & $m_{b}(\mathrm{GeV})$ & $A$ & $B\left(\mathrm{GeV}^{-1}\right)$ \\
\hline 0.0 & 5.00 & 15.4 & -0.0559 \\
0.25 & 6.16 & 5.84 & -0.0152 \\
0.50 & 6.60 & 2.55 & -0.0145 \\
\hline
\end{tabular}

Table I.b Fitted Parameters for $d \sigma / d p_{T}^{2} / d x_{F}$ for: $0<p_{T}<20 \mathrm{GeV}$ at $\sqrt{s}=200 \mathrm{GeV}$.

\begin{tabular}{|l|c|c|c|}
\hline$x_{F}$ & $m_{b}(\mathrm{GeV})$ & $A$ & $B\left(\mathrm{GeV}^{-1}\right)$ \\
\hline 0.0 & 5.53 & 7.16 & -0.363 \\
0.25 & 6.34 & 4.06 & -0.274 \\
0.50 & 6.14 & 0.383 & -0.246 \\
\hline
\end{tabular}

Table I.c Fitted Parameters for $d \sigma / d p_{T}^{2} / d x_{F}$ for: $0<p_{T}<30 \mathrm{GeV}$ at LHC Collider Energy.

\begin{tabular}{|l|c|c|c|}
\hline$x_{F}$ & $m_{b}(\mathrm{GeV})$ & $A$ & $B\left(\mathrm{GeV}^{-1}\right)$ \\
\hline 0.0 & 5.35 & 14.2 & -0.0873 \\
0.25 & 5.96 & 5.58 & -0.0318 \\
0.50 & 6.81 & 2.46 & -0.0376 \\
\hline
\end{tabular}


Table I.d Fitted Parameters for $d \sigma / d p_{T}^{2} / d x_{F}$ for: $0<p_{T}<15 \mathrm{GeV}$ at $\sqrt{s}=120 \mathrm{GeV}$.

\begin{tabular}{|l|c|c|c|}
\hline$x_{F}$ & $m_{b}(\mathrm{GeV})$ & $A$ & $B\left(\mathrm{GeV}^{-1}\right)$ \\
\hline 0.0 & 6.76 & 6.80 & -0.516 \\
0.25 & 7.95 & 4.58 & -0.440 \\
0.50 & 7.74 & 1.02 & -0.408 \\
\hline
\end{tabular}

In Fig. 3 and Table I.b, we provide analogous results for the SSC fixed target energy of $\sqrt{s}=200 \mathrm{GeV}$. The $x_{F}$ dependence is less dramatic at this energy.

In Fig. 4 and Table I.c, we present results for the LHC collider energy of $\sqrt{s}=$ 15.4 TeV. The $x_{F}$ dependence at this energy shows a steep decrease from $x_{F}=0$ to 0.25 , followed by a more gradual decrease from 0.25 to 0.5 , as we saw above at $\sqrt{s}=40 \mathrm{TeV}$. Fitted values for the LHC cross section at $\sqrt{s}=120 \mathrm{GeV}$ are provided in Table I.d

We acknowledge valuable communications with Stanley Wojcicki. We are grateful to W. James Stirling for providing a copy of the Martin, Roberts, and Stirling parton densities. This work was supported in part by the U.S. Department of Energy, Division of High Energy Physics, Contract W-31-109-ENG-38.

\section{References}

1. P. Nason, S. Dawson, and R. K. Ellis, Nucl. Phys. B303, 607 (1988); B327, 49 (1989).

2. W. Beenakker, H. Kuijf, W. L. van Neerven, and J. Smith, Phys. Rev. D40, 54 (1989); W. Beenakker, W. L. van Neerven, R. Meng, G. Schuler, and J. Smith, Nucl. Phys. B351, 507 (1991).

3. A. D. Martin, R. G. Roberts, and W. J. Stirling, Phys. Lett. B306, 145 (1993), Erratum-ibid. B309, 492 (1993).

4. E. L. Berger, and R. Meng, Phys. Rev. D46, 169 (1992).

5. E. L. Berger and R. Meng, Phys.Lett. B304, 318 (1993); E. L. Berger and R. Meng, in the Proc. DPF92, Fermilab, 1992, edited by C. Albright, P. Kasper, R. Raja, and J. Yoh, vol. 2, p. 954; E. L. Berger, R. Meng, and J.-W. Qiu, in the Proc. XXVI Int. 
Conf. on High Energy Physics, Dallas, 1992, edited by J. R. Sanford, vol. 1, p. 853;

E. L. Berger, R. Meng, and W.-K. Tung, Phys. Rev. D46, 1859 (1992).

6. J.C. Collins and R.K. Ellis, Nucl. Phys. B360, 3 (1991); S. Catani, M. Ciafaloni, and F. Hautmann, Nucl. Phys. B366, 135 (1991); E.M. Levin et al., Sov. J. Nucl. Phys. 54, 867 (1991).

\section{Figure Captions}

Fig. 1. a) The calculated cross section $\sigma(p p \rightarrow b \bar{b} X)$ at order $\alpha_{s}^{3}$ in QCD is shown as a function of $\sqrt{s}$ for $100<\sqrt{s}<2000 \mathrm{GeV}$. The solid line is obtained from the parton densities of MRS $D_{o}^{\prime}$ ("regular" gluon), and the dashed curve from the MRS $D_{-}^{\prime}$ ("singular" gluon). b) As in (a), but for $\sqrt{s} \geq 2 \mathrm{TeV}$.

Fig. 2. The calculated order $\alpha_{s}^{3} \mathrm{QCD}$ differential cross section $d \sigma / d x_{F} / d p_{T}^{2}$ vs. $p_{T}$ for the SSC collider energy $\sqrt{s}=40 \mathrm{TeV}$ is shown (solid curves) for (a) $x_{F}=0$; (b) $x_{F}=0.25$; (c) $x_{F}=0.50$. Phenomenological parametrizations fitted to the theoretical calculations are presented as the histograms.

Fig. 3. As in Fig. 2 but for the proposed SSC fixed target energy $\sqrt{s}=200 \mathrm{GeV}$.

Fig. 4. As in Fig. 2 but for the LHC collider energy $\sqrt{s}=15.4 \mathrm{TeV}$. 


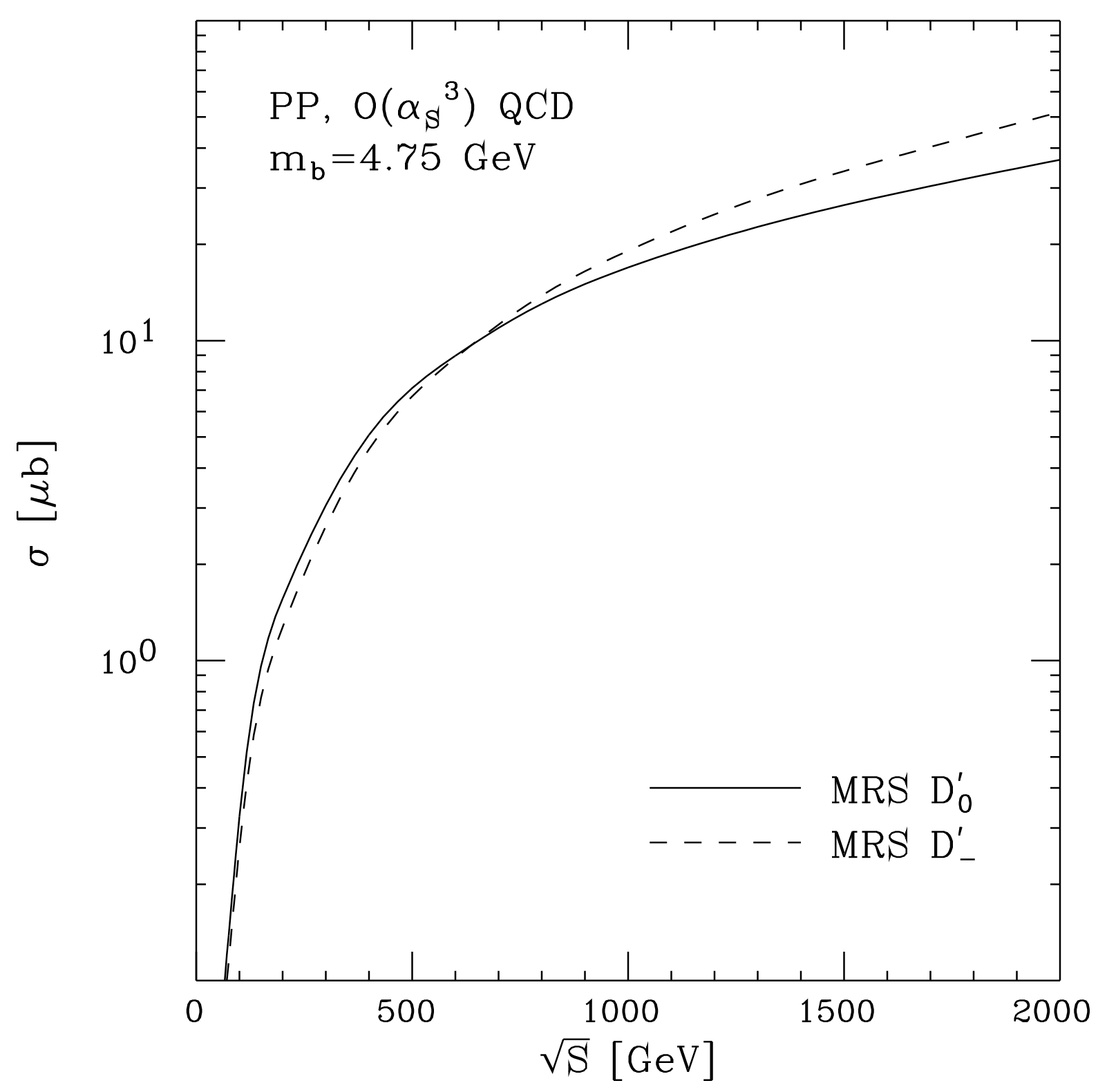

Figure 1(a) 


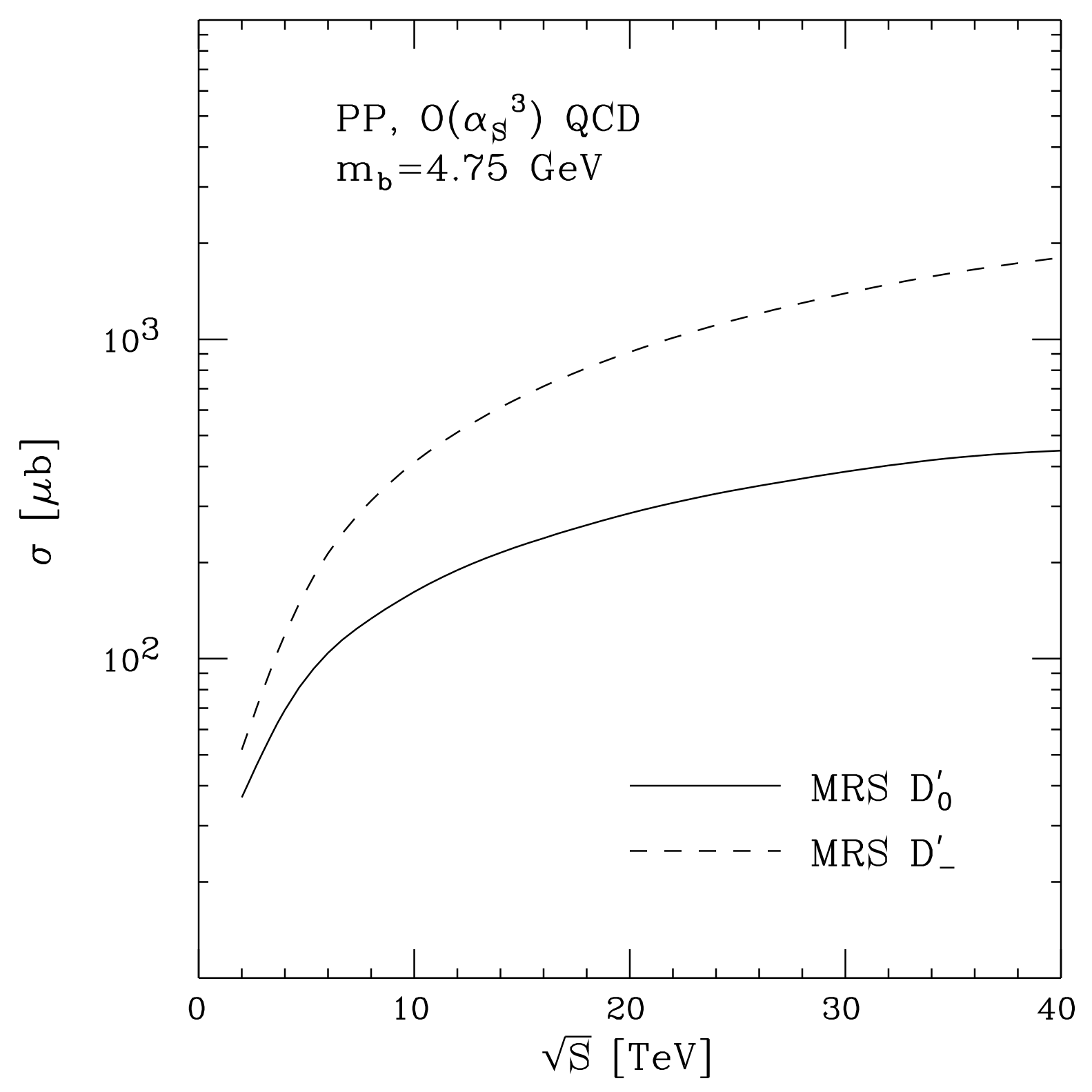

Figure 1(b) 


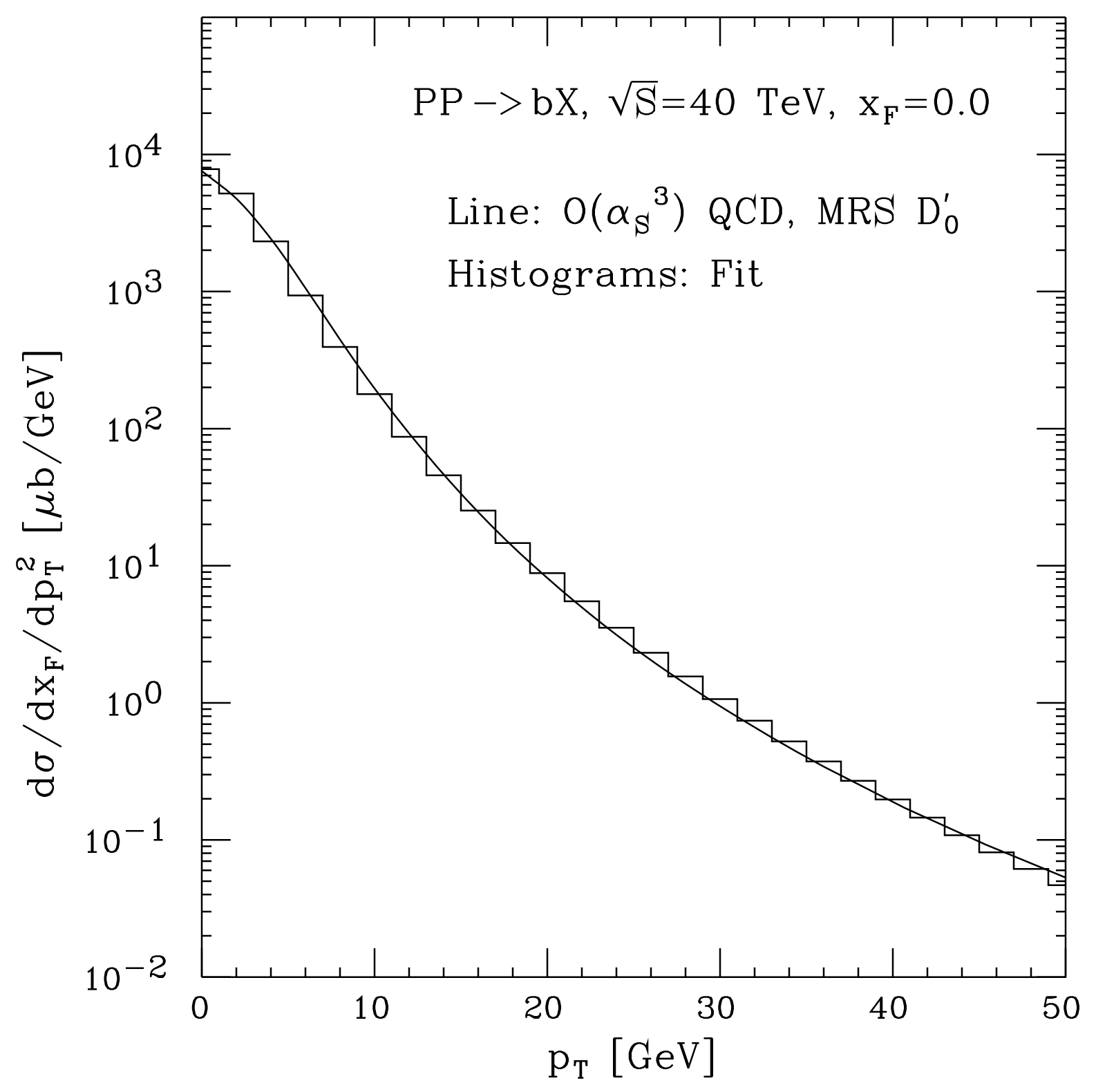

Figure 2(a) 


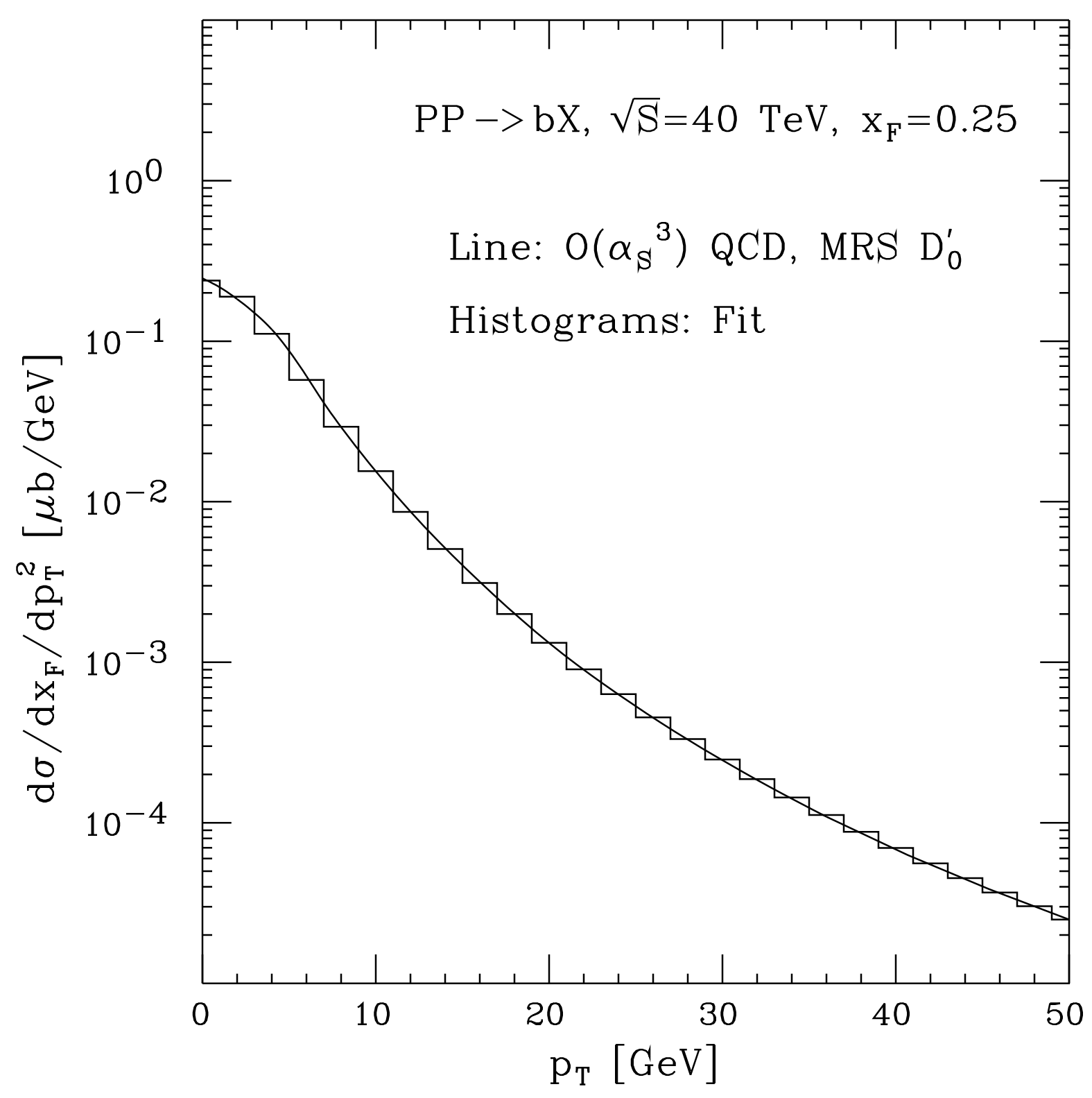

Figure 2(b) 


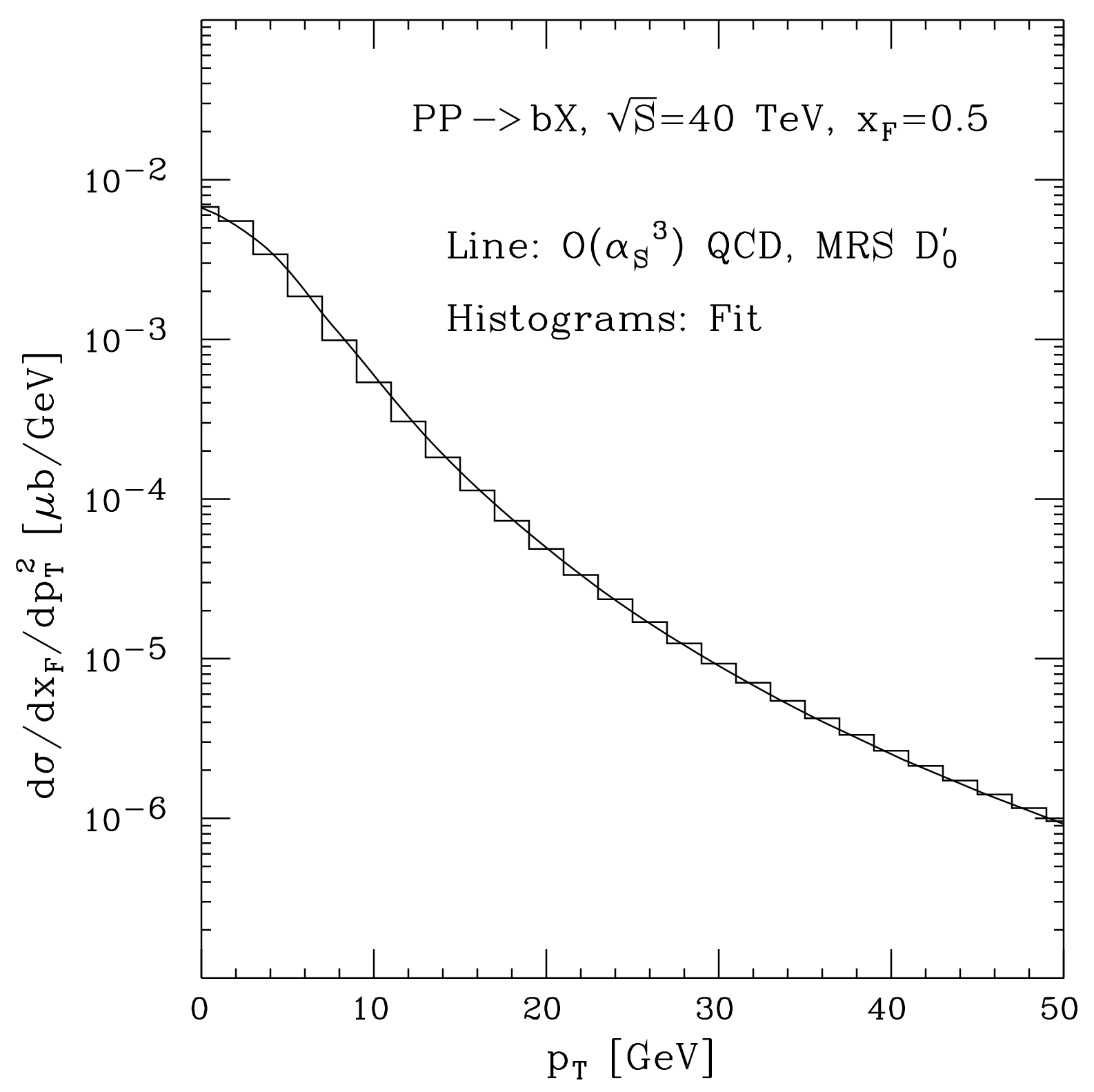

Figure 2(c) 


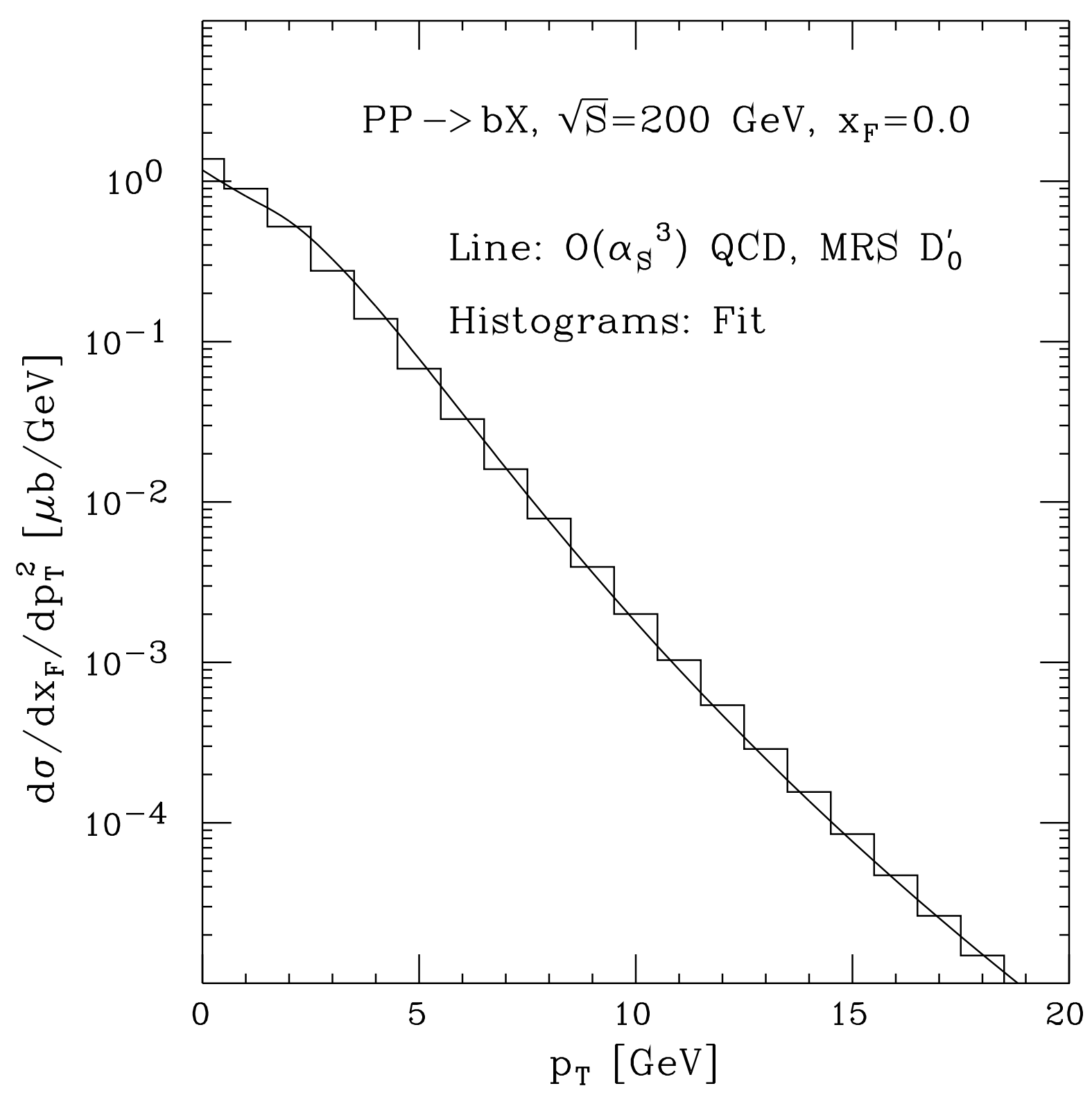

Figure 3(a) 


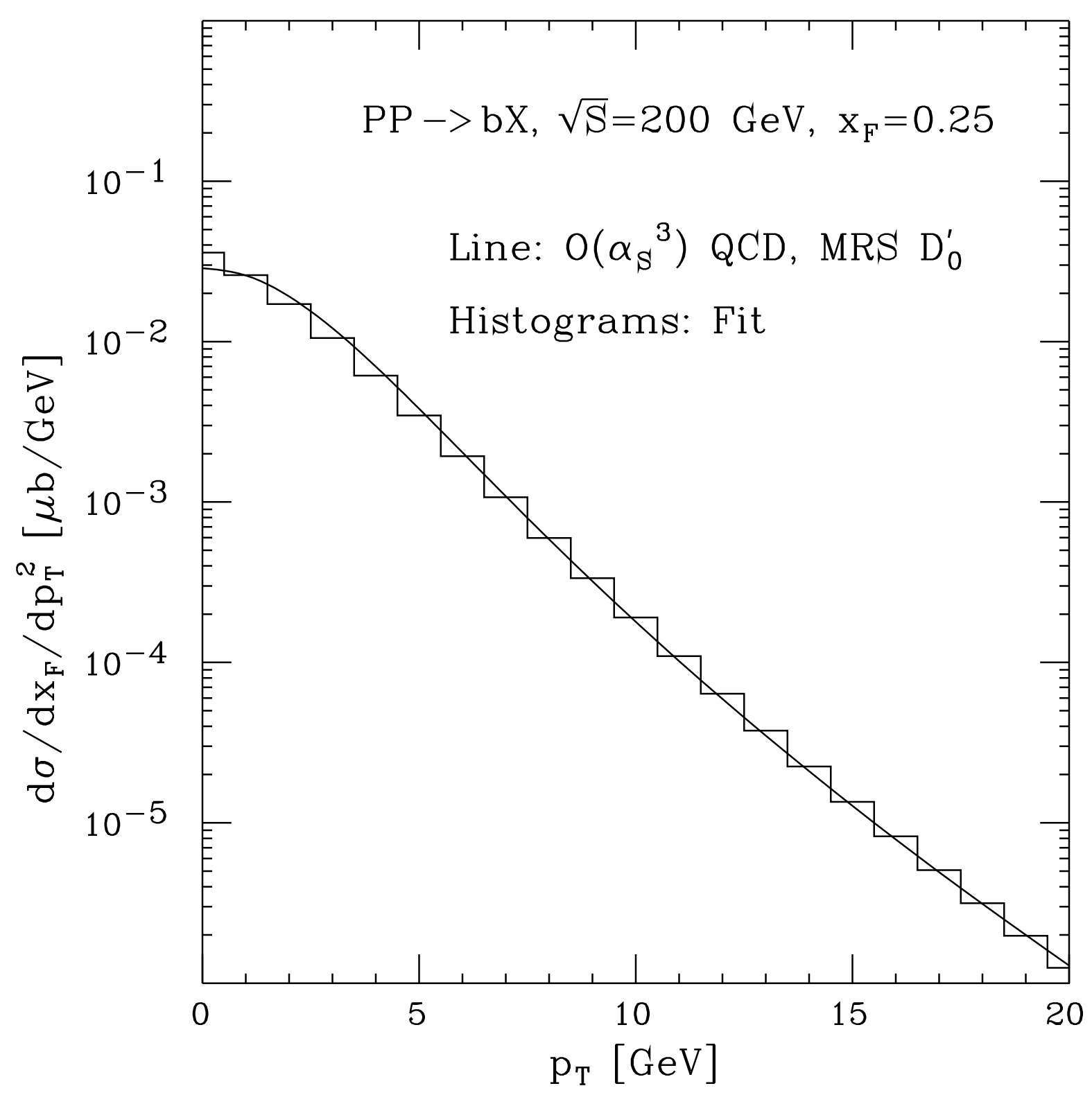

Figure 3(b) 


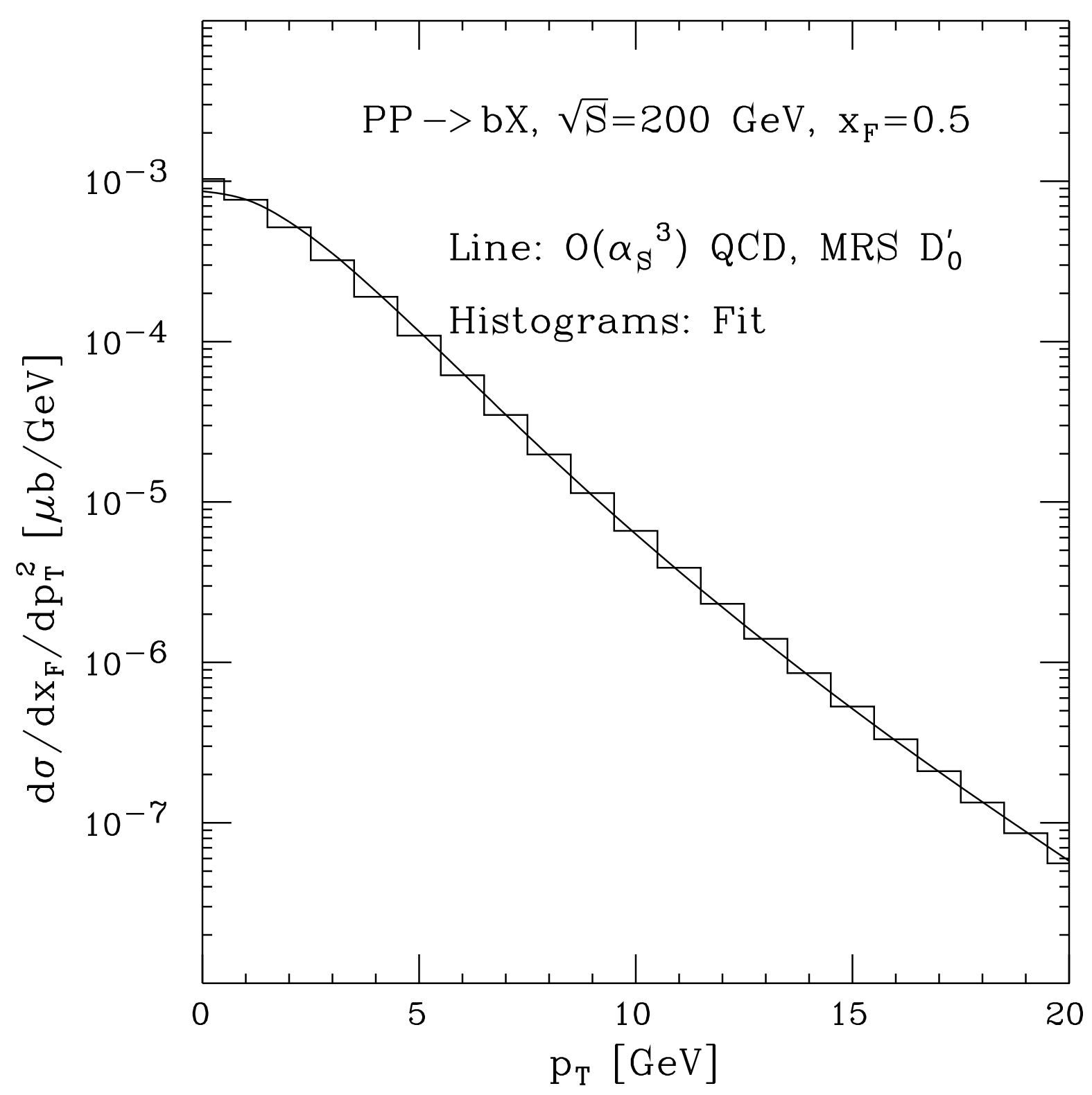

Figure 3(c) 


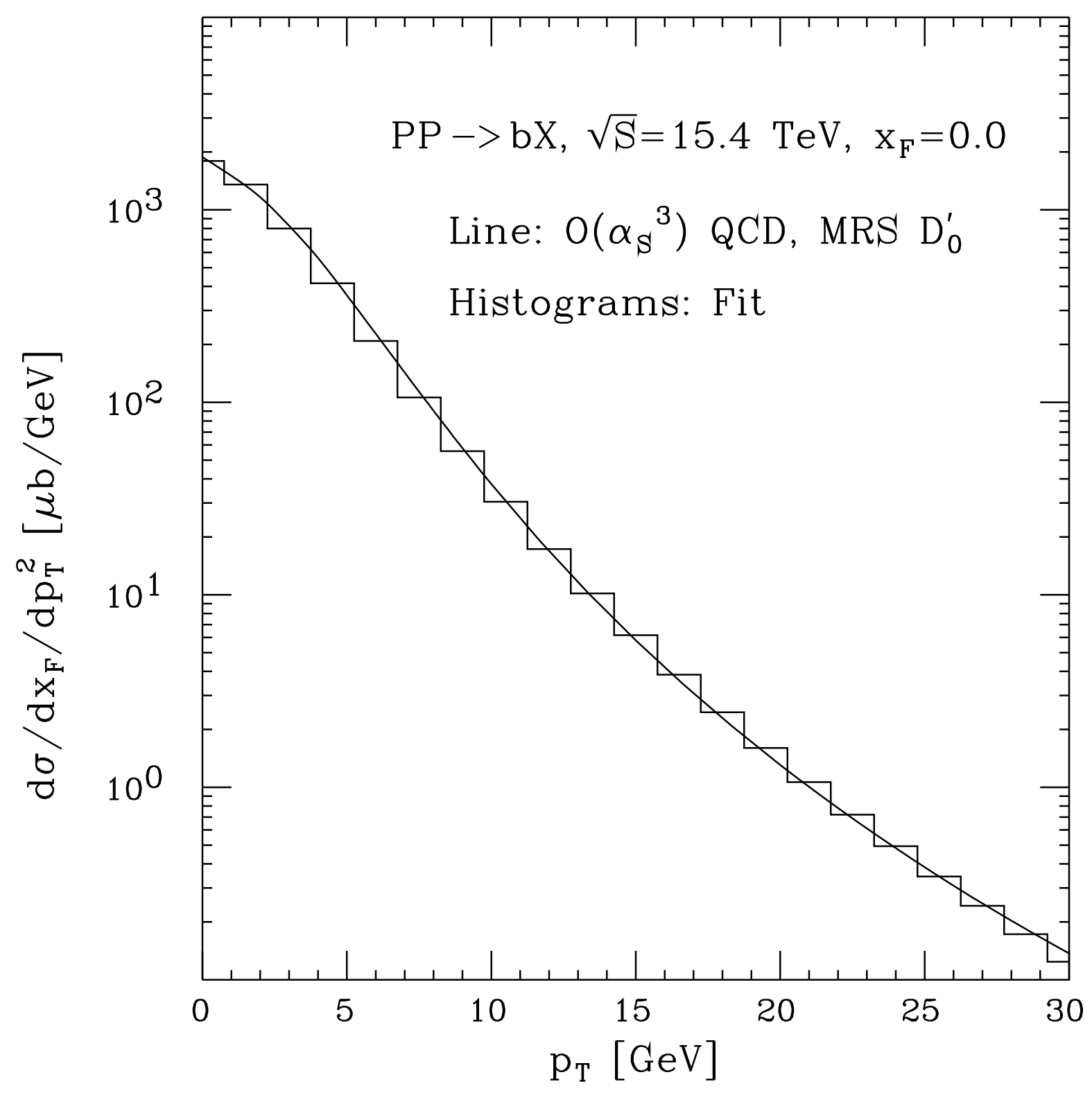

Figure 4(a) 


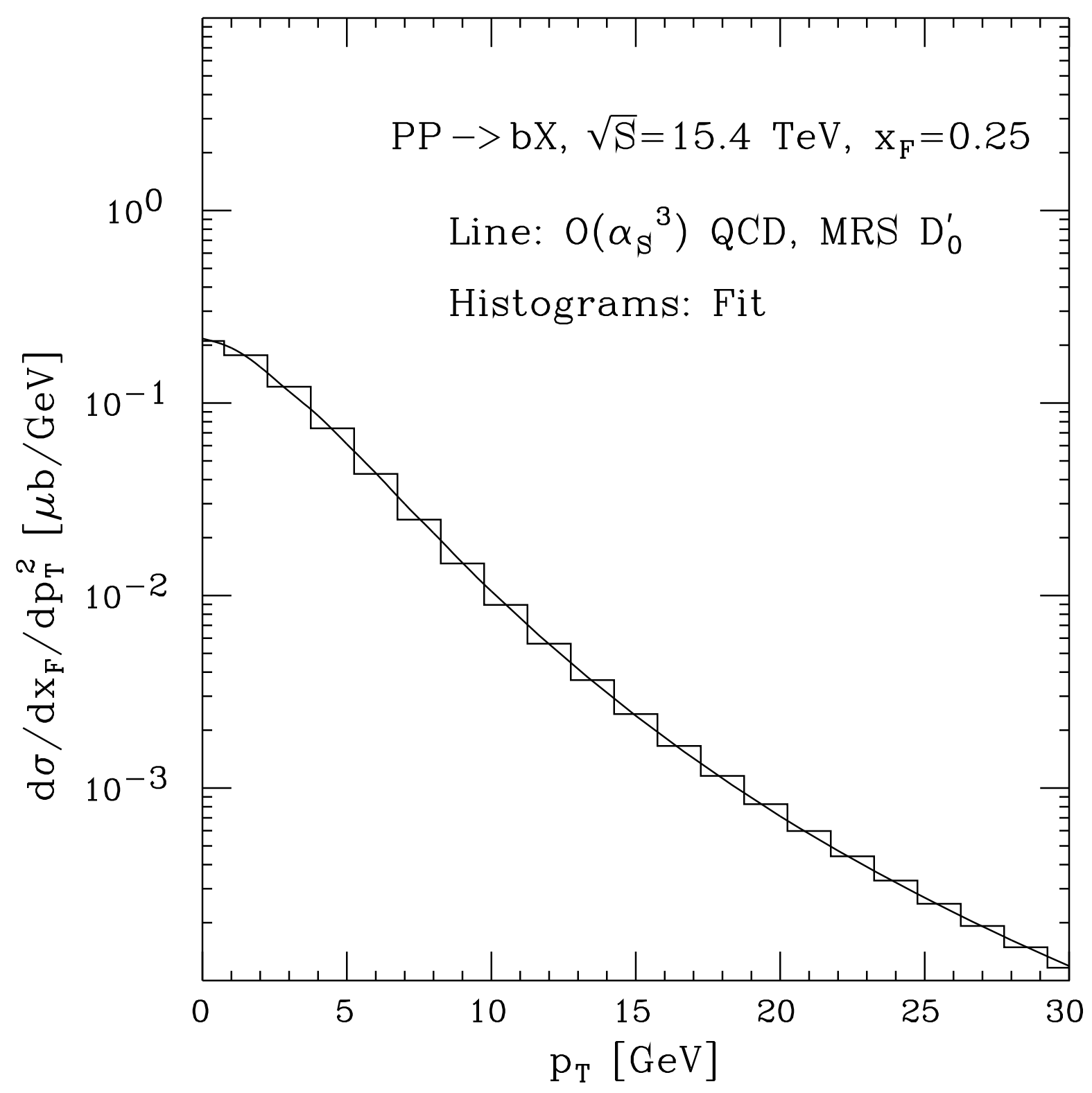

Figure 4(b) 


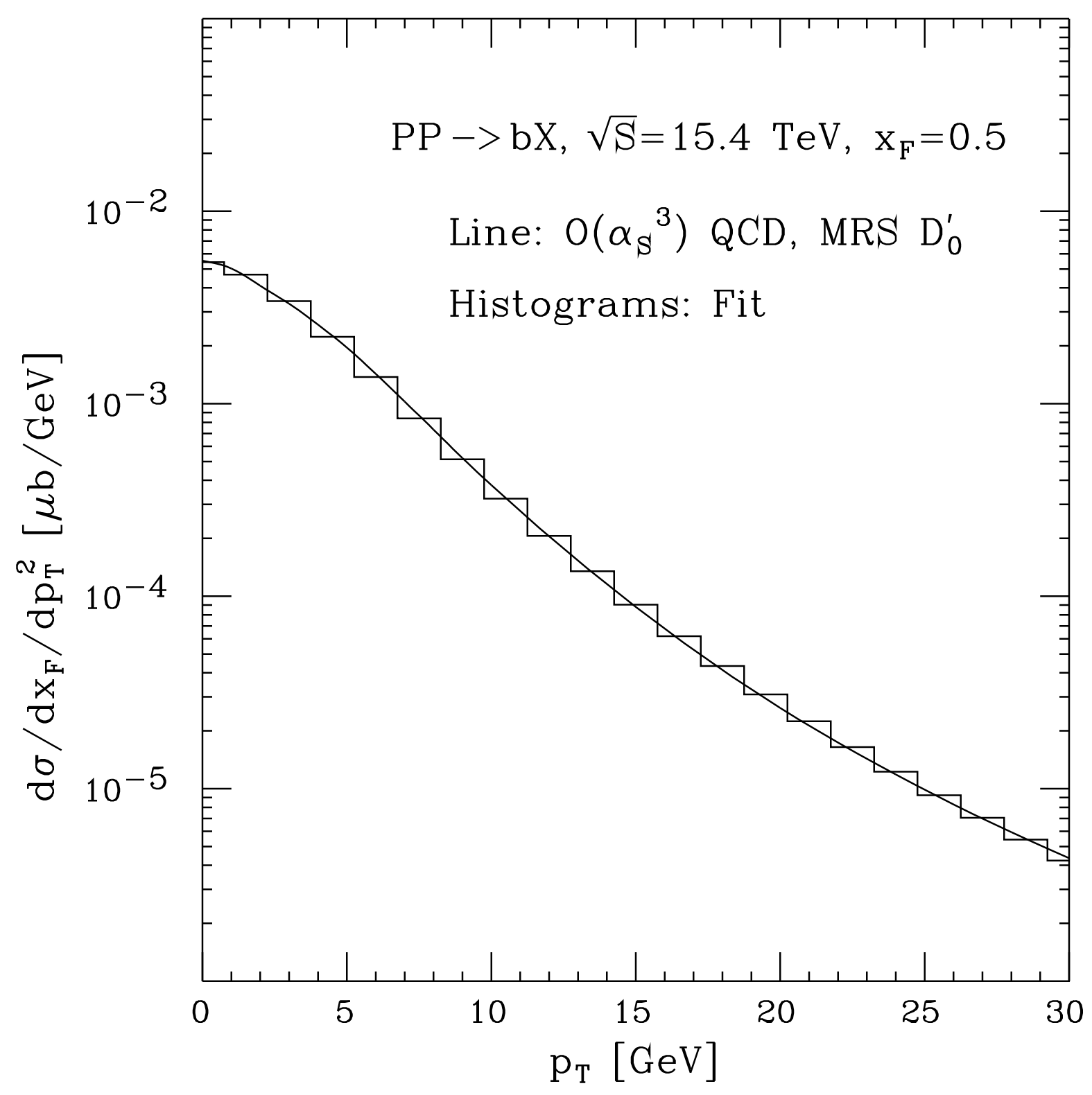

Figure 4(c) 